\title{
Editorial Note: Emerging Multimedia Technology for Multimedia-centric IoT
}

Multimedia tools and applications gratefully acknowledges the editorial work of the scholars listed below on the special issue entitled, "Emerging Multimedia Technology for Multimediacentric IoT."

Of 28 papers submitted to this issue, 13 were eventually accepted after a stringent peerreview process.

\section{Bryan (Byung-Gyu) Kim (Corresponding Guest Editor)}

Sookmyung Women's University, Seoul, Rep. of Korea

bg.kim@ieee.org

\section{Naveen Chilamkurti}

La Trobe University, Melbourne Campus, Australia

N.Chilamkurti@latrobe.edu.au

\section{Debi Prosad Dogra}

School of Electrical Sciences, IIT Bhubaneswar, India

dpdogra@iitbbs.ac.in

\section{Kostas Psannis}

University of Macedonia, Thessaloniki, Greece

kpsannis@uom.gr 